\title{
The reciprocal sums of the Fibonacci 3-subsequences
}

\section{Andrew YZ Wang ${ }^{*}$ and Fan Zhang}

"Correspondence:

yzwang@uestc.edu.cn

School of Mathematical Sciences,

University of Electronic Science and

Technology of China, Chengdu,

611731, P.R. China

\begin{abstract}
A Fibonacci 3-subsequence is a subsequence of the type $F_{n}, F_{n+3}, F_{n+6}, \ldots$, where $F_{k}$ denotes the $k$ th Fibonacci number. In this article, we investigate the reciprocal sums of the Fibonacci 3-subsequences and obtain several interesting families of identities involving the Fibonacci numbers.
\end{abstract}

MSC: $11 \mathrm{~B} 39$

Keywords: Fibonacci 3-subsequence; reciprocal sum; floor function

\section{Introduction}

The Fibonacci sequence is defined by the linear recurrence relation

$$
F_{n}=F_{n-1}+F_{n-2} \text { for } n \geq 2,
$$

where $F_{n}$ is called the $n$th Fibonacci number with $F_{0}=0$ and $F_{1}=1$. There exists a simple and nonobvious formula for the Fibonacci numbers,

$$
F_{n}=\frac{1}{\sqrt{5}}\left(\frac{1+\sqrt{5}}{2}\right)^{n}-\frac{1}{\sqrt{5}}\left(\frac{1-\sqrt{5}}{2}\right)^{n} .
$$

The Fibonacci sequence plays an important role in the theory and applications of mathematics, and its various properties have been investigated by many authors: see [1-5].

In recent years, there has been an increasing interest in studying the reciprocal sums of the Fibonacci numbers. For example, Elsner, Shimomura, and Shiokawa [6-9] investigated the algebraic relations for reciprocal sums of the Fibonacci numbers. Ohtsuka and Nakamura [10] studied the partial infinite sums of the reciprocal Fibonacci numbers. They established the following results, where $L \cdot\rfloor$ denotes the floor function.

Theorem 1.1 For all $n \geq 2$,

$$
\left\lfloor\left(\sum_{k=n}^{\infty} \frac{1}{F_{k}}\right)^{-1}\right\rfloor= \begin{cases}F_{n-2} & \text { if } n \text { is even } \\ F_{n-2}-1 & \text { if } n \text { is odd } .\end{cases}
$$

\section{Springer}


Theorem 1.2 For all $n \geq 1$,

$$
\left\lfloor\left(\sum_{k=n}^{\infty} \frac{1}{F_{k}^{2}}\right)^{-1}\right\rfloor= \begin{cases}F_{n} F_{n-1}-1 & \text { if } n \text { is even }, \\ F_{n} F_{n-1} & \text { if } n \text { is odd } .\end{cases}
$$

Recently, Wang and Wen [11] considered the partial finite sum of the reciprocal Fibonacci numbers and strengthened Theorem 1.1 and Theorem 1.2 to the finite sum case.

\section{Theorem 1.3}

(i) For all $n \geq 4$,

$$
\left\lfloor\left(\sum_{k=n}^{2 n} \frac{1}{F_{k}}\right)^{-1}\right\rfloor=F_{n-2}
$$

(ii) If $m \geq 3$ and $n \geq 2$, then

$$
\left\lfloor\left(\sum_{k=n}^{m n} \frac{1}{F_{k}}\right)^{-1}\right\rfloor= \begin{cases}F_{n-2} & \text { if } n \text { is even } \\ F_{n-2}-1 & \text { if } n \text { is odd. }\end{cases}
$$

Theorem 1.4 For all $m \geq 2$ and $n \geq 1$, we have

$$
\left\lfloor\left(\sum_{k=n}^{m n} \frac{1}{F_{k}^{2}}\right)^{-1}\right\rfloor= \begin{cases}F_{n} F_{n-1}-1 & \text { if } n \text { is even }, \\ F_{n} F_{n-1} & \text { if } n \text { is odd. }\end{cases}
$$

Furthermore, the present authors [12] studied the reciprocal sums of even and odd terms in the Fibonacci sequence and obtained the following main results.

Theorem 1.5 We have

$$
\left\lfloor\left(\sum_{k=n}^{m n} \frac{1}{F_{2 k}}\right)^{-1}\right\rfloor= \begin{cases}F_{2 n-1} & \text { if } m=2 \text { and } n \geq 3 \\ F_{2 n-1}-1 & \text { if } m \geq 3 \text { and } n \geq 1\end{cases}
$$

Theorem 1.6 For all $n \geq 1$ and $m \geq 2$, we have

$$
\left\lfloor\left(\sum_{k=n}^{m n} \frac{1}{F_{2 k-1}}\right)^{-1}\right\rfloor=F_{2 n-2} .
$$

Theorem 1.7 If $n \geq 1$ and $m \geq 2$, then

$$
\left\lfloor\left(\sum_{k=n}^{m n} \frac{1}{F_{2 k}^{2}}\right)^{-1}\right\rfloor=F_{4 n-2}-1 .
$$

Theorem 1.8 For all $n \geq 1$ and $m \geq 2$, we have

$$
\left\lfloor\left(\sum_{k=n}^{m n} \frac{1}{F_{2 k-1}^{2}}\right)^{-1}\right\rfloor=F_{4 n-4} .
$$


In this article, applying elementary methods, we investigate the reciprocal sums of the Fibonacci 3-subsequences, by which we mean the subsequences of the type $F_{n}, F_{n+3}$, $F_{n+6}, \ldots$ and obtain several interesting families of identities involving the Fibonacci numbers.

\section{Main results I: the reciprocal sums}

We first present several well-known results on Fibonacci numbers, which will be used throughout the article. The detailed proofs can be found in [5].

Lemma 2.1 For any positive integers $a$ and $b$, we have

$$
F_{a} F_{b}+F_{a+1} F_{b+1}=F_{a+b+1} .
$$

As a consequence of (2.1), we have the following conclusion.

Corollary 2.2 If $n \geq 1$, then

$$
\begin{aligned}
& F_{2 n+1}=F_{n-1} F_{n+1}+F_{n} F_{n+2}, \\
& F_{2 n+1}=F_{n+1} F_{n+2}-F_{n-1} F_{n} .
\end{aligned}
$$

The following interesting identity concerning the Fibonacci numbers plays a central role in the proofs of our main results.

Lemma 2.3 Assume that $a, b, c$ are given nonnegative integers with $a \geq b$. For $n \geq a+c$, we have

$$
F_{n+a} F_{n-a-c}-F_{n+b} F_{n-b-c}=(-1)^{n-a-c+1} F_{a+b+c} F_{a-b} .
$$

Proof We proceed by induction on $n$. It is clearly true for $n=a+c$. Assuming that the result holds for any integer $n \geq a+c$, we show that the same is true for $n+1$.

First, it is easy to check that

$$
\begin{aligned}
F_{n-1+a} F_{n-a-c}+F_{n+a} F_{n-1-a-c} & =F_{n-1+a} F_{n-a-c}+F_{n+a}\left(F_{n+1-a-c}-F_{n-a-c}\right) \\
& =F_{n-1+a} F_{n-a-c}+F_{n+a} F_{n+1-a-c}-F_{n+a} F_{n-a-c} \\
& =F_{2 n-c}-F_{n+a} F_{n-a-c},
\end{aligned}
$$

where the last equality follows from (2.1).

Now we have

$$
\begin{aligned}
F_{n+1+a} F_{n+1-a-c} & =\left(F_{n-1+a}+F_{n+a}\right)\left(F_{n-1-a-c}+F_{n-a-c}\right) \\
& =F_{n-1+a} F_{n-a-c}+F_{n+a} F_{n-1-a-c}+F_{n+a} F_{n-a-c}+F_{n-1+a} F_{n-1-a-c} \\
& =F_{2 n-c}+F_{n-1+a} F_{n-1-a-c} .
\end{aligned}
$$

Similarly, we get $F_{n+1+b} F_{n+1-b-c}=F_{2 n-c}+F_{n-1+b} F_{n-1-b-c}$. 
Therefore, by the induction hypothesis, we arrive at

$$
\begin{aligned}
F_{n+1+a} F_{n+1-a-c}-F_{n+1+b} F_{n+1-b-c} & =F_{(n-1)+a} F_{(n-1)-a-c}-F_{(n-1)+b} F_{(n-1)-b-c} \\
& =(-1)^{n-a-c} F_{a+b+c} F_{a-b},
\end{aligned}
$$

which completes the induction on $n$.

Before introducing our main results, we first establish an inequality.

Lemma 2.4 If $n \geq 8$, then

$$
F_{n-2} F_{n} F_{n+1}>2 F_{2 n+1} \text {. }
$$

Proof It follows from (2.3) that

$$
F_{n+1} F_{n+2}>F_{2 n+1} \text {. }
$$

Since $n \geq 8$, we have

$$
\begin{aligned}
F_{n-2} F_{n}-2 F_{n+2} & =F_{n-2} F_{n}-2\left(F_{n}+F_{n+1}\right) \\
& =F_{n-2} F_{n}-2\left(F_{n-1}+2 F_{n}\right) \\
& =F_{n}\left(F_{n-2}-4\right)-2 F_{n-1} \\
& >2 F_{n}-2 F_{n-1} \\
& >0
\end{aligned}
$$

Thus, $F_{n-2} F_{n} F_{n+1}>2 F_{n+2} F_{n+1}>2 F_{2 n+1}$.

Theorem 2.5 For all $n \geq 2$,

$$
\left\lfloor\left(\sum_{k=n}^{2 n} \frac{1}{F_{3 k}}\right)^{-1}\right\rfloor=2 F_{3 n-2} .
$$

Proof Setting $n=3 k, a=1, b=0$, and $c=2$ in (2.4), we obtain

$$
F_{3 k+1} F_{3 k-3}-F_{3 k} F_{3 k-2}=(-1)^{3 k-2} 2=(-1)^{k} 2 .
$$

It is straightforward to check that, for $k \geq 1$,

$$
\begin{aligned}
\frac{1}{2 F_{3 k-2}}-\frac{1}{F_{3 k}}-\frac{1}{2 F_{3 k+1}} & =\frac{F_{3 k} F_{3 k+1}-2 F_{3 k-2} F_{3 k+1}-F_{3 k} F_{3 k-2}}{2 F_{3 k-2} F_{3 k} F_{3 k+1}} \\
& =\frac{F_{3 k+1}\left(F_{3 k}-2 F_{3 k-2}\right)-F_{3 k} F_{3 k-2}}{2 F_{3 k-2} F_{3 k} F_{3 k+1}} \\
& =\frac{F_{3 k+1} F_{3 k-3}-F_{3 k} F_{3 k-2}}{2 F_{3 k-2} F_{3 k} F_{3 k+1}}=\frac{(-1)^{k}}{F_{3 k-2} F_{3 k} F_{3 k+1}},
\end{aligned}
$$

where the last equality follows from (2.7). 
Therefore, we get that

$$
\sum_{k=n}^{2 n} \frac{1}{F_{3 k}}=\frac{1}{2 F_{3 n-2}}-\frac{1}{2 F_{6 n+1}}+\sum_{k=n}^{2 n} \frac{(-1)^{k-1}}{F_{3 k-2} F_{3 k} F_{3 k+1}} .
$$

If $n$ is even, it is clear that

$$
\sum_{k=n}^{2 n} \frac{(-1)^{k-1}}{F_{3 k-2} F_{3 k} F_{3 k+1}}<0
$$

thus,

$$
\sum_{k=n}^{2 n} \frac{1}{F_{3 k}}<\frac{1}{2 F_{3 n-2}}
$$

Now we consider the case where $n$ is odd. It follows from (2.5) and the condition $n \geq 3$ that

$$
F_{3 n-2} F_{3 n} F_{3 n+1}>2 F_{6 n+1} \text {, }
$$

from which we derive

$$
\begin{aligned}
\sum_{k=n}^{2 n} \frac{1}{F_{3 k}} & =\frac{1}{2 F_{3 n-2}}-\frac{1}{2 F_{6 n+1}}+\frac{1}{F_{3 n-2} F_{3 n} F_{3 n+1}}+\sum_{k=n+1}^{2 n} \frac{(-1)^{k-1}}{F_{3 k-2} F_{3 k} F_{3 k+1}} \\
& <\frac{1}{2 F_{3 n-2}}-\frac{1}{2 F_{6 n+1}}+\frac{1}{F_{3 n-2} F_{3 n} F_{3 n+1}} \\
& <\frac{1}{2 F_{3 n-2}} .
\end{aligned}
$$

Hence, we always have

$$
\sum_{k=n}^{2 n} \frac{1}{F_{3 k}}<\frac{1}{2 F_{3 n-2}}
$$

irrespective of the parity of $n$.

If we let $n=3 k, a=1, b=0$, and $c=1$ in (2.4), then

$$
F_{3 k+1} F_{3 k-2}-F_{3 k} F_{3 k-1}=(-1)^{k-1} \text {. }
$$

By elementary manipulations and (2.10) we deduce that, for $k \geq 1$,

$$
\begin{aligned}
\frac{1}{2 F_{3 k-2}+1}-\frac{1}{F_{3 k}}-\frac{1}{2 F_{3 k+1}+1} & =\frac{(-1)^{k} 4-2 F_{3 k-2}-\left(2 F_{3 k+1}+1\right)}{\left(2 F_{3 k-2}+1\right) F_{3 k}\left(2 F_{3 k+1}+1\right)} \\
& =\frac{(-1)^{k} 4-2 F_{3 k-2}}{\left(2 F_{3 k-2}+1\right) F_{3 k}\left(2 F_{3 k+1}+1\right)}-\frac{1}{\left(2 F_{3 k-2}+1\right) F_{3 k}} \\
& <-\frac{1}{\left(2 F_{3 k-2}+1\right) F_{3 k}},
\end{aligned}
$$


which implies that

$$
\begin{aligned}
\sum_{k=n}^{2 n} \frac{1}{F_{3 k}} & >\frac{1}{2 F_{3 n-2}+1}-\frac{1}{2 F_{6 n+1}+1}+\sum_{k=n}^{2 n} \frac{1}{\left(2 F_{3 k-2}+1\right) F_{3 k}} \\
& >\frac{1}{2 F_{3 n-2}+1}-\frac{1}{2 F_{6 n+1}+1}+\frac{1}{\left(2 F_{3 n-2}+1\right) F_{3 n}} .
\end{aligned}
$$

It follows from (2.2) that

$$
\begin{aligned}
2 F_{6 n+1} & =2 F_{3 n-1} F_{3 n+1}+2 F_{3 n} F_{3 n+2} \\
& >2 F_{3 n-2} F_{3 n}+F_{3 n} \\
& =\left(2 F_{3 n-2}+1\right) F_{3 n},
\end{aligned}
$$

from which we get that

$$
\sum_{k=n}^{2 n} \frac{1}{F_{3 k}}>\frac{1}{2 F_{3 n-2}+1} .
$$

Combining (2.9) and (2.11), we have

$$
\frac{1}{2 F_{3 n-2}+1}<\sum_{k=n}^{2 n} \frac{1}{F_{3 k}}<\frac{1}{2 F_{3 n-2}},
$$

which yields the desired identity.

We now study a generalization of Theorem 2.5 and start with an inequality.

Lemma 2.6 If $m \geq 3$ and $n \geq 3$, then

$$
\frac{1}{F_{n-2} F_{n} F_{n+1}}-\frac{1}{F_{n+1} F_{n+3} F_{n+4}}>\frac{1}{2 F_{m n+1}} .
$$

Proof Letting $a=b=n-1$ in (2.1), we obtain $F_{n} F_{n}<F_{2 n-1}$. If we set $a=2 n-1$ and $b=n+1$ in (2.1), then $F_{2 n-1} F_{n+1}<F_{3 n+1}$. Therefore,

$$
F_{n} F_{n} F_{n+1}<F_{3 n+1},
$$

from which we derive that

$$
\begin{aligned}
\frac{1}{F_{n-2} F_{n} F_{n+1}}-\frac{1}{F_{n+1} F_{n+3} F_{n+4}}-\frac{1}{2 F_{m n+1}} & \geq \frac{1}{F_{n-2} F_{n} F_{n+1}}-\frac{1}{F_{n+1} F_{n+3} F_{n+4}}-\frac{1}{2 F_{3 n+1}} \\
& >\frac{1}{F_{n-2} F_{n} F_{n+1}}-\frac{1}{F_{n+1} F_{n+3} F_{n+4}}-\frac{1}{F_{n} F_{n} F_{n+1}} \\
& =\frac{F_{n-1}}{F_{n-2} F_{n} F_{n} F_{n+1}}-\frac{F_{n-1}}{F_{n-1} F_{n+1} F_{n+3} F_{n+4}} \\
& >0,
\end{aligned}
$$

which completes the proof. 
Theorem 2.7 If $m \geq 3$ and $n \geq 1$, then

$$
\left\lfloor\left(\sum_{k=n}^{m n} \frac{1}{F_{3 k}}\right)^{-1}\right\rfloor= \begin{cases}2 F_{3 n-2} & \text { if } n \text { is even, } \\ 2 F_{3 n-2}-1 & \text { if } n \text { is odd. }\end{cases}
$$

Proof Applying the same argument as in the proof of Theorem 2.5, it is easy to see that if $n$ is even, then

$$
\frac{1}{2 F_{3 n-2}+1}<\sum_{k=n}^{m n} \frac{1}{F_{3 k}}<\frac{1}{2 F_{3 n-2}}
$$

and thus the statement is true when $n$ is even. We now concentrate ourselves on the case where $n$ is odd.

Invoking (2.10), a direct calculation shows that, for $k \geq 1$,

$$
\begin{aligned}
\frac{1}{2 F_{3 k-2}-1}-\frac{1}{F_{3 k}}-\frac{1}{2 F_{3 k+1}-1} & =\frac{2 F_{3 k}\left(F_{3 k+1}-F_{3 k-2}\right)-\left(2 F_{3 k-2}-1\right)\left(2 F_{3 k+1}-1\right)}{\left(2 F_{3 k-2}-1\right) F_{3 k}\left(2 F_{3 k+1}-1\right)} \\
& =\frac{4\left(F_{3 k} F_{3 k-1}-F_{3 k+1} F_{3 k-2}\right)+2 F_{3 k-2}+2 F_{3 k+1}-1}{\left(2 F_{3 k-2}-1\right) F_{3 k}\left(2 F_{3 k+1}-1\right)} \\
& =\frac{(-1)^{k} 4+2 F_{3 k-2}+2 F_{3 k+1}-1}{\left(2 F_{3 k-2}-1\right) F_{3 k}\left(2 F_{3 k+1}-1\right)} \\
& >0
\end{aligned}
$$

Hence, we arrive at

$$
\sum_{k=n}^{m n} \frac{1}{F_{3 k}}<\frac{1}{2 F_{3 n-2}-1}-\frac{1}{2 F_{3 m n+1}-1}<\frac{1}{2 F_{3 n-2}-1} .
$$

Employing (2.8) and the fact that $n$ is odd, we conclude that

$$
\begin{aligned}
\sum_{k=n}^{m n} \frac{1}{F_{3 k}} & =\frac{1}{2 F_{3 n-2}}-\frac{1}{2 F_{3 m n+1}}+\sum_{k=n}^{m n} \frac{(-1)^{k-1}}{F_{3 k-2} F_{3 k} F_{3 k+1}} \\
& >\frac{1}{2 F_{3 n-2}}-\frac{1}{2 F_{3 m n+1}}+\frac{1}{F_{3 n-2} F_{3 n} F_{3 n+1}}-\frac{1}{F_{3 n+1} F_{3 n+3} F_{3 n+4}} \\
& >\frac{1}{2 F_{3 n-2}}
\end{aligned}
$$

where the last inequality follows from Lemma 2.6.

It follows from (2.14) and (2.15) that if $m \geq 3$ and $n$ is odd, then

$$
\frac{1}{2 F_{3 n-2}}<\sum_{k=n}^{m n} \frac{1}{F_{3 k}}<\frac{1}{2 F_{3 n-2}-1}
$$

from which the desired identity follows immediately.

Applying a similar analysis to the subsequences $\left\{F_{3 k+1}\right\}$ and $\left\{F_{3 k+2}\right\}$, we obtain the following results, whose proofs are omitted here. 
Theorem 2.8 For all $n \geq 1$,

$$
\left\lfloor\left(\sum_{k=n}^{2 n} \frac{1}{F_{3 k+1}}\right)^{-1}\right\rfloor=2 F_{3 n-1} .
$$

Theorem 2.9 If $m \geq 3$ and $n \geq 1$, then

$$
\left\lfloor\left(\sum_{k=n}^{m n} \frac{1}{F_{3 k+1}}\right)^{-1}\right\rfloor= \begin{cases}2 F_{3 n-1}-1 & \text { if } n \text { is even } \\ 2 F_{3 n-1} & \text { if } n \text { is odd } .\end{cases}
$$

Theorem 2.10 For all $n \geq 1$,

$$
\left\lfloor\left(\sum_{k=n}^{2 n} \frac{1}{F_{3 k+2}}\right)^{-1}\right\rfloor=2 F_{3 n}
$$

Theorem 2.11 If $m \geq 3$ and $n \geq 1$, then

$$
\left\lfloor\left(\sum_{k=n}^{m n} \frac{1}{F_{3 k+2}}\right)^{-1}\right\rfloor= \begin{cases}2 F_{3 n} & \text { if } n \text { is even } \\ 2 F_{3 n}-1 & \text { if } n \text { is odd } .\end{cases}
$$

\section{Main results II: the reciprocal square sums}

In the rest of the article, we study the reciprocal square sums of the Fibonacci 3-subsequences.

Lemma 3.1 Let $a$ and $b$ be two given integers with $a \geq b \geq 0$. For all $n \geq a$, we have

$$
F_{n+a}^{2} F_{n-a}^{2}-F_{n+b}^{2} F_{n-b}^{2}=F_{a+b} F_{a-b}\left(F_{a}^{2}+(-1)^{a-b} F_{b}^{2}+(-1)^{n-a+1} 2 F_{n}^{2}\right) .
$$

Proof Employing (2.4), we derive that

$$
\begin{aligned}
F_{n+a}^{2} F_{n-a}^{2}-F_{n+b}^{2} F_{n-b}^{2} & =\left(F_{n+a} F_{n-a}-F_{n+b} F_{n-b}\right)\left(F_{n+a} F_{n-a}+F_{n+b} F_{n-b}\right) \\
& =(-1)^{n-a+1} F_{a+b} F_{a-b}\left(2 F_{n}^{2}+F_{n+a} F_{n-a}-F_{n}^{2}+F_{n+b} F_{n-b}-F_{n}^{2}\right) \\
& =(-1)^{n-a+1} F_{a+b} F_{a-b}\left(2 F_{n}^{2}+(-1)^{n-a+1} F_{a}^{2}+(-1)^{n-b+1} F_{b}^{2}\right) \\
& =F_{a+b} F_{a-b}\left(F_{a}^{2}+(-1)^{a-b} F_{b}^{2}+(-1)^{n-a+1} 2 F_{n}^{2}\right) .
\end{aligned}
$$

The proof is complete.

Lemma 3.2 If $n \geq 1$, we have

$$
F_{6 n+3}^{2}-F_{6 n}^{2}>F_{3 n}^{2}\left(F_{3 n+3}^{2}-F_{3 n}^{2}+1\right)
$$

Proof First, it is easy to see that

$$
F_{6 n+3}^{2}-F_{6 n}^{2}=\left(F_{6 n+3}+F_{6 n}\right)\left(F_{6 n+3}-F_{6 n}\right)=4 F_{6 n+1} F_{6 n+2} .
$$


Similarly, we have

$$
F_{3 n+3}^{2}-F_{3 n}^{2}=4 F_{3 n+1} F_{3 n+2}
$$

It follows from (2.1) that $F_{6 n+1}>F_{3 n}^{2}+1$ and $F_{6 n+2}>F_{3 n+1} F_{3 n+2}+1$. Therefore,

$$
F_{6 n+1} F_{6 n+2}>F_{3 n}^{2} F_{3 n+1} F_{3 n+2}+F_{3 n}^{2} .
$$

The desired result follows immediately from (3.3), (3.4), and (3.5).

Lemma 3.3 For $n \geq 1$, we have

$$
\frac{8 F_{3 n}^{2}-16}{\left(F_{3 n}^{2}-F_{3 n-3}^{2}\right) F_{3 n}^{2}\left(F_{3 n+3}^{2}-F_{3 n}^{2}\right)}-\frac{8 F_{3 n+3}^{2}+16}{\left(F_{3 n+3}^{2}-F_{3 n}^{2}\right) F_{3 n+3}^{2}\left(F_{3 n+6}^{2}-F_{3 n+3}^{2}\right)}>\frac{1}{F_{3 n}^{2} F_{3 n+3}^{2}} .
$$

Proof It is easy to see that $8 F_{3 n}^{2}-16>F_{3 n}^{2}-F_{3 n-3}^{2}$ and $8 F_{3 n+3}^{2}+16<F_{3 n+6}^{2}-F_{3 n+3}^{2}$, so

$$
\frac{8 F_{3 n}^{2}-16}{\left(F_{3 n}^{2}-F_{3 n-3}^{2}\right) F_{3 n}^{2}\left(F_{3 n+3}^{2}-F_{3 n}^{2}\right)}>\frac{1}{F_{3 n}^{2}\left(F_{3 n+3}^{2}-F_{3 n}^{2}\right)}
$$

and

$$
\frac{1}{\left(F_{3 n+3}^{2}-F_{3 n}^{2}\right) F_{3 n+3}^{2}}>\frac{8 F_{3 n+3}^{2}+16}{\left(F_{3 n+3}^{2}-F_{3 n}^{2}\right) F_{3 n+3}^{2}\left(F_{3 n+6}^{2}-F_{3 n+3}^{2}\right)}
$$

Hence,

$$
\begin{gathered}
\frac{8 F_{3 n}^{2}-16}{\left(F_{3 n}^{2}-F_{3 n-3}^{2}\right) F_{3 n}^{2}\left(F_{3 n+3}^{2}-F_{3 n}^{2}\right)}-\frac{8 F_{3 n+3}^{2}+16}{\left(F_{3 n+3}^{2}-F_{3 n}^{2}\right) F_{3 n+3}^{2}\left(F_{3 n+6}^{2}-F_{3 n+3}^{2}\right)} \\
>\frac{1}{F_{3 n}^{2}\left(F_{3 n+3}^{2}-F_{3 n}^{2}\right)}-\frac{1}{\left(F_{3 n+3}^{2}-F_{3 n}^{2}\right) F_{3 n+3}^{2}}=\frac{1}{F_{3 n}^{2} F_{3 n+3}^{2}},
\end{gathered}
$$

which completes the proof.

We now present the first reciprocal square sum of the Fibonacci 3-subsequence.

Theorem 3.4 If $n \geq 2$ and $m \geq 2$, then

$$
\left\lfloor\left(\sum_{k=n}^{m n} \frac{1}{F_{3 k}^{2}}\right)^{-1}\right\rfloor= \begin{cases}F_{3 n}^{2}-F_{3 n-3}^{2} & \text { if } n \text { is even }, \\ F_{3 n}^{2}-F_{3 n-3}^{2}-1 & \text { if } n \text { is odd } .\end{cases}
$$

Proof We first consider the case where $n$ is even. Applying (3.1), by a direct calculation we get that, for $k \geq 1$,

$$
\begin{aligned}
& \frac{1}{F_{3 k}^{2}-F_{3 k-3}^{2}}-\frac{1}{F_{3 k}^{2}}-\frac{1}{F_{3 k+3}^{2}-F_{3 k}^{2}} \\
& =\frac{F_{3 k}^{2}\left(F_{3 k+3}^{2}-2 F_{3 k}^{2}+F_{3 k-3}^{2}\right)-\left(F_{3 k}^{2}-F_{3 k-3}^{2}\right)\left(F_{3 k+3}^{2}-F_{3 k}^{2}\right)}{\left(F_{3 k}^{2}-F_{3 k-3}^{2}\right) F_{3 k}^{2}\left(F_{3 k+3}^{2}-F_{3 k}^{2}\right)}
\end{aligned}
$$




$$
\begin{aligned}
& =\frac{F_{3 k+3}^{2} F_{3 k-3}^{2}-F_{3 k}^{4}}{\left(F_{3 k}^{2}-F_{3 k-3}^{2}\right) F_{3 k}^{2}\left(F_{3 k+3}^{2}-F_{3 k}^{2}\right)} \\
& =\frac{16+(-1)^{3 k} 8 F_{3 k}^{2}}{\left(F_{3 k}^{2}-F_{3 k-3}^{2}\right) F_{3 k}^{2}\left(F_{3 k+3}^{2}-F_{3 k}^{2}\right)},
\end{aligned}
$$

from which we get

$$
\sum_{k=n}^{m n} \frac{1}{F_{3 k}^{2}}=\frac{1}{F_{3 n}^{2}-F_{3 n-3}^{2}}-\frac{1}{F_{3 m n+3}^{2}-F_{3 m n}^{2}}-8 \sum_{k=n}^{m n} \frac{(-1)^{3 k} F_{3 k}^{2}+2}{\left(F_{3 k}^{2}-F_{3 k-3}^{2}\right) F_{3 k}^{2}\left(F_{3 k+3}^{2}-F_{3 k}^{2}\right)} .
$$

Since $n$ is even, it is easy to see that

$$
\sum_{k=n}^{m n} \frac{(-1)^{3 k} F_{3 k}^{2}+2}{\left(F_{3 k}^{2}-F_{3 k-3}^{2}\right) F_{3 k}^{2}\left(F_{3 k+3}^{2}-F_{3 k}^{2}\right)}>0
$$

which implies that

$$
\sum_{k=n}^{m n} \frac{1}{F_{3 k}^{2}}<\frac{1}{F_{3 n}^{2}-F_{3 n-3}^{2}}-\frac{1}{F_{3 m n+3}^{2}-F_{3 m n}^{2}}<\frac{1}{F_{3 n}^{2}-F_{3 n-3}^{2}} .
$$

A similar manipulation yields that, for $k \geq 1$,

$$
\begin{aligned}
\frac{1}{F_{3 k}^{2}-F_{3 k-3}^{2}+1}-\frac{1}{F_{3 k}^{2}}-\frac{1}{F_{3 k+3}^{2}-F_{3 k}^{2}+1} & =\frac{F_{3 k-3}^{2}-F_{3 k+3}^{2}+15+(-1)^{3 k} 8 F_{3 k}^{2}}{\left(F_{3 k}^{2}-F_{3 k-3}^{2}+1\right) F_{3 k}^{2}\left(F_{3 k+3}^{2}-F_{3 k}^{2}+1\right)} \\
& \leq-\frac{F_{3 k+3}^{2}-F_{3 k-3}^{2}-15-8 F_{3 k}^{2}}{\left(F_{3 k}^{2}-F_{3 k-3}^{2}+1\right) F_{3 k}^{2}\left(F_{3 k+3}^{2}-F_{3 k}^{2}+1\right)} \\
& <-\frac{1}{F_{3 k}^{2}\left(F_{3 k+3}^{2}-F_{3 k}^{2}+1\right)},
\end{aligned}
$$

where the last inequality follows from the easily checked fact

$$
F_{3 k+3}^{2}-F_{3 k-3}^{2}-15-8 F_{3 k}^{2}>F_{3 k}^{2}-F_{3 k-3}^{2}+1 .
$$

Now we have

$$
\begin{aligned}
\sum_{k=n}^{m n} \frac{1}{F_{3 k}^{2}} & >\frac{1}{F_{3 n}^{2}-F_{3 n-3}^{2}+1}-\frac{1}{F_{3 m n+3}^{2}-F_{3 m n}^{2}+1}+\sum_{k=n}^{m n} \frac{1}{F_{3 k}^{2}\left(F_{3 k+3}^{2}-F_{3 k}^{2}+1\right)} \\
& >\frac{1}{F_{3 n}^{2}-F_{3 n-3}^{2}+1}-\frac{1}{F_{3 m n+3}^{2}-F_{3 m n}^{2}+1}+\frac{1}{F_{3 n}^{2}\left(F_{3 n+3}^{2}-F_{3 n}^{2}+1\right)} \\
& \geq \frac{1}{F_{3 n}^{2}-F_{3 n-3}^{2}+1}+\frac{1}{F_{3 n}^{2}\left(F_{3 n+3}^{2}-F_{3 n}^{2}+1\right)}-\frac{1}{F_{6 n+3}^{2}-F_{6 n}^{2}+1} \\
& >\frac{1}{F_{3 n}^{2}-F_{3 n-3}^{2}+1},
\end{aligned}
$$

where the last inequality follows from (3.2). 
Combining (3.8) and (3.9), we get that

$$
\frac{1}{F_{3 n}^{2}-F_{3 n-3}^{2}+1}<\sum_{k=n}^{m n} \frac{1}{F_{3 k}^{2}}<\frac{1}{F_{3 n}^{2}-F_{3 n-3}^{2}}
$$

which means that the statement is true when $n$ is even.

We now consider the case where $n$ is odd. It is not hard to derive that

$$
\begin{aligned}
& \frac{1}{F_{3 k}^{2}-F_{3 k-3}^{2}-1}-\frac{1}{F_{3 k}^{2}}-\frac{1}{F_{3 k+3}^{2}-F_{3 k}^{2}-1} \\
& \quad=\frac{(-1)^{k} 8 F_{3 k}^{2}+F_{3 k+3}^{2}-F_{3 k-3}^{2}+15}{\left(F_{3 k}^{2}-F_{3 k-3}^{2}-1\right) F_{3 k}^{2}\left(F_{3 k+3}^{2}-F_{3 k}^{2}-1\right)} \\
& \quad>0
\end{aligned}
$$

where the last inequality follows from the fact that $F_{3 k+3}=3 F_{3 k}+2 F_{3 k-1}$.

Therefore, we arrive at

$$
\sum_{k=n}^{m n} \frac{1}{F_{3 k}^{2}}<\frac{1}{F_{3 n}^{2}-F_{3 n-3}^{2}-1}-\frac{1}{F_{3 m n+3}^{2}-F_{3 m n}^{2}-1}<\frac{1}{F_{3 n}^{2}-F_{3 n-3}^{2}-1}
$$

Invoking (3.7) and Lemma 3.3, we derive that if $n$ is odd, then

$$
\begin{aligned}
\sum_{k=n}^{m n} \frac{1}{F_{3 k}^{2}=} & \frac{1}{F_{3 n}^{2}-F_{3 n-3}^{2}}-\frac{1}{F_{3 m n+3}^{2}-F_{3 m n}^{2}} \\
& +\sum_{k=n}^{m n} \frac{(-1)^{k-1} 8 F_{3 k}^{2}-16}{\left(F_{3 k}^{2}-F_{3 k-3}^{2}\right) F_{3 k}^{2}\left(F_{3 k+3}^{2}-F_{3 k}^{2}\right)} \\
> & \frac{1}{F_{3 n}^{2}-F_{3 n-3}^{2}}+\frac{8 F_{3 n}^{2}-16}{\left(F_{3 n}^{2}-F_{3 n-3}^{2}\right) F_{3 n}^{2}\left(F_{3 n+3}^{2}-F_{3 n}^{2}\right)} \\
& -\frac{8 F_{3 n+3}^{2}+16}{\left(F_{3 n+3}^{2}-F_{3 n}^{2}\right) F_{3 n+3}^{2}\left(F_{3 n+6}^{2}-F_{3 n+3}^{2}\right)}-\frac{1}{F_{6 n+3}^{2}-F_{6 n}^{2}} \\
> & \frac{1}{F_{3 n}^{2}-F_{3 n-3}^{2}}+\frac{1}{F_{3 n}^{2} F_{3 n+3}^{2}}-\frac{1}{F_{6 n+3}^{2}-F_{6 n}^{2}} \\
= & \frac{1}{F_{3 n}^{2}-F_{3 n-3}^{2}}+\frac{1}{F_{3 n}^{2} F_{3 n+3}^{2}}-\frac{1}{4 F_{6 n+1} F_{6 n+2}} \\
> & \frac{1}{F_{3 n}^{2}-F_{3 n-3}^{2}},
\end{aligned}
$$

where the last inequality follows from the fact that $F_{6 n+2}>F_{3 n} F_{3 n+3}$ and $2 F_{6 n+1}>F_{6 n+2}$. It follows from (3.10) and (3.11) that

$$
\frac{1}{F_{3 n}^{2}-F_{3 n-3}^{2}}<\sum_{k=n}^{m n} \frac{1}{F_{3 k}^{2}}<\frac{1}{F_{3 n}^{2}-F_{3 n-3}^{2}-1}
$$

which yields the desired result.

For the subsequences $\left\{F_{3 k+1}\right\}$ and $\left\{F_{3 k+2}\right\}$, we have similar results. 
Theorem 3.5 If $n \geq 1$ and $m \geq 2$, then

$$
\left\lfloor\left(\sum_{k=n}^{m n} \frac{1}{F_{3 k+1}^{2}}\right)^{-1}\right\rfloor= \begin{cases}F_{3 n+1}^{2}-F_{3 n-2}^{2}-1 & \text { if } n \text { is even }, \\ F_{3 n+1}^{2}-F_{3 n-2}^{2} & \text { if } n \text { is odd } .\end{cases}
$$

Theorem 3.6 If $n \geq 1$ and $m \geq 2$, then

$$
\left\lfloor\left(\sum_{k=n}^{m n} \frac{1}{F_{3 k+2}^{2}}\right)^{-1}\right\rfloor= \begin{cases}F_{3 n+2}^{2}-F_{3 n-1}^{2} & \text { if } n \text { is even }, \\ F_{3 n+2}^{2}-F_{3 n-1}^{2}-1 & \text { if } n \text { is odd } .\end{cases}
$$

Remark The proof of Theorem 3.5 is similar to that of Theorem 3.4 and is omitted here. Since the telescoping technique for the proof of Theorem 3.6 is very different from that for Theorem 3.4, we give a detailed proof of Theorem 3.6 in the next section.

\section{Proof of Theorem 3.6}

We first present a preliminary result, which plays a central role in the later proof.

Lemma 4.1 For all $n \geq 1$, we have

$$
\begin{aligned}
2\left(F_{6 n+5}^{2}-F_{6 n+2}^{2}\right) & >F_{3 n+2}^{2} F_{3 n+5}^{2} \\
& >\left(F_{3 n+2}^{2}-F_{3 n-1}^{2}+1\right)\left(F_{3 n+5}^{2}-F_{3 n+2}^{2}+1\right) .
\end{aligned}
$$

Proof The second inequality is obvious, so we concentrate ourselves on the first one. It is easy to see that

$$
2\left(F_{6 n+5}^{2}-F_{6 n+2}^{2}\right)=2 \cdot 4 F_{6 n+3} F_{6 n+4}>4 F_{6 n+4}^{2} .
$$

Employing (2.1), we get $F_{6 n+4}=F_{3 n+2}\left(F_{3 n+1}+F_{3 n+3}\right)$; therefore,

$$
F_{6 n+4}^{2}=F_{3 n+2}^{2}\left(F_{3 n+1}+F_{3 n+3}\right)^{2} .
$$

It follows from $2 F_{3 n+1}>F_{3 n+2}=F_{3 n+5}-2 F_{3 n+3}$ that $2\left(F_{3 n+1}+F_{3 n+3}\right)>F_{3 n+5}$; thus,

$$
4\left(F_{3 n+1}+F_{3 n+3}\right)^{2}>F_{3 n+5}^{2} .
$$

Combining (4.1), (4.2), and (4.3) yields the desired result.

Proof of Theorem 3.6 We first consider the case where $n$ is even. Employing (3.1) again, by a direct calculation we deduce that, for $k \geq 1$,

$$
\begin{aligned}
& \frac{1}{F_{3 k+2}^{2}-F_{3 k-1}^{2}}-\frac{1}{F_{3 k+2}^{2}}-\frac{1}{F_{3 k+5}^{2}-F_{3 k+2}^{2}} \\
& \quad=\frac{F_{3 k+2}^{2}\left(F_{3 k+5}^{2}-2 F_{3 k+2}^{2}+F_{3 k-1}^{2}\right)-\left(F_{3 k+2}^{2}-F_{3 k-1}^{2}\right)\left(F_{3 k+5}^{2}-F_{3 k+2}^{2}\right)}{\left(F_{3 k+2}^{2}-F_{3 k-1}^{2}\right) F_{3 k+2}^{2}\left(F_{3 k+5}^{2}-F_{3 k+2}^{2}\right)} \\
& \quad=\frac{F_{3 k-1}^{2} F_{3 k+5}^{2}-F_{3 k+2}^{4}}{\left(F_{3 k+2}^{2}-F_{3 k-1}^{2}\right) F_{3 k+2}^{2}\left(F_{3 k+5}^{2}-F_{3 k+2}^{2}\right)} \\
& =\frac{16+(-1)^{k} 8 F_{3 k+2}^{2}}{\left(F_{3 k+2}^{2}-F_{3 k-1}^{2}\right) F_{3 k+2}^{2}\left(F_{3 k+5}^{2}-F_{3 k+2}^{2}\right)}
\end{aligned}
$$


from which we obtain

$$
\begin{aligned}
\sum_{k=n}^{m n} \frac{1}{F_{3 k+2}^{2}}= & \frac{1}{F_{3 n+2}^{2}-F_{3 n-1}^{2}}-\frac{1}{F_{3 m n+5}^{2}-F_{3 m n+2}^{2}} \\
& -8 \sum_{k=n}^{m n} \frac{(-1)^{k} F_{3 k+2}^{2}+2}{\left(F_{3 k+2}^{2}-F_{3 k-1}^{2}\right) F_{3 k+2}^{2}\left(F_{3 k+5}^{2}-F_{3 k+2}^{2}\right)}
\end{aligned}
$$

Since $n$ is even, it is easy to see that

$$
\sum_{k=n}^{m n} \frac{(-1)^{k} F_{3 k+2}^{2}+2}{\left(F_{3 k+2}^{2}-F_{3 k-1}^{2}\right) F_{3 k+2}^{2}\left(F_{3 k+5}^{2}-F_{3 k+2}^{2}\right)}>0
$$

which implies that

$$
\sum_{k=n}^{m n} \frac{1}{F_{3 k+2}^{2}}<\frac{1}{F_{3 n+2}^{2}-F_{3 n-1}^{2}} .
$$

Similarly, we can derive that, for $k \geq 1$,

$$
\begin{aligned}
& \frac{1}{F_{3 k+2}^{2}-F_{3 k-1}^{2}+1}-\frac{1}{F_{3 k+2}^{2}}-\frac{1}{F_{3 k+5}^{2}-F_{3 k+2}^{2}+1} \\
& \quad=\frac{F_{3 k-1}^{2}-F_{3 k+5}^{2}+15+(-1)^{k} 8 F_{3 k+2}^{2}}{\left(F_{3 k+2}^{2}-F_{3 k-1}^{2}+1\right) F_{3 k+2}^{2}\left(F_{3 k+5}^{2}-F_{3 k+2}^{2}+1\right)} \\
& \quad<0 .
\end{aligned}
$$

We now have

$$
\begin{aligned}
\sum_{k=n}^{m n} \frac{1}{F_{3 k+2}^{2}}= & \frac{1}{F_{3 n+2}^{2}-F_{3 n-1}^{2}+1}-\frac{1}{F_{3 m n+5}^{2}-F_{3 m n+2}^{2}+1} \\
& +\sum_{k=n}^{m n} \frac{F_{3 k+5}^{2}-F_{3 k-1}^{2}-15-(-1)^{k} 8 F_{3 k+2}^{2}}{\left(F_{3 k+2}^{2}-F_{3 k-1}^{2}+1\right) F_{3 k+2}^{2}\left(F_{3 k+5}^{2}-F_{3 k+2}^{2}+1\right)} \\
> & \frac{1}{F_{3 n+2}^{2}-F_{3 n-1}^{2}+1}+\frac{F_{3 n+5}^{2}-F_{3 n-1}^{2}-15-8 F_{3 n+2}^{2}}{\left(F_{3 n+2}^{2}-F_{3 n-1}^{2}+1\right) F_{3 n+2}^{2}\left(F_{3 n+5}^{2}-F_{3 n+2}^{2}+1\right)} \\
& -\frac{1}{F_{6 n+5}^{2}-F_{6 n+2}^{2}+1} \\
> & \frac{1}{F_{3 n+2}^{2}-F_{3 n-1}^{2}+1}+\frac{2}{\left(F_{3 n+2}^{2}-F_{3 n-1}^{2}+1\right)\left(F_{3 n+5}^{2}-F_{3 n+2}^{2}+1\right)} \\
& -\frac{1}{F_{6 n+5}^{2}-F_{6 n+2}^{2}+1} \\
> & \frac{1}{F_{3 n+2}^{2}-F_{3 n-1}^{2}+1}
\end{aligned}
$$

where the last inequality follows from Lemma 4.1. 
Combining (4.5) and (4.6) yields that

$$
\frac{1}{F_{3 n+2}^{2}-F_{3 n-1}^{2}+1}<\sum_{k=n}^{m n} \frac{1}{F_{3 k+2}^{2}}<\frac{1}{F_{3 n+2}^{2}-F_{3 n-1}^{2}}
$$

which shows that the statement is true when $n$ is even.

Next we turn to consider the case where $n$ is odd. It is not hard to derive that

$$
\begin{aligned}
& \frac{1}{F_{3 k+2}^{2}-F_{3 k-1}^{2}-1}-\frac{1}{F_{3 k+2}^{2}}-\frac{1}{F_{3 k+5}^{2}-F_{3 k+2}^{2}-1} \\
& \quad=\frac{(-1)^{k} 8 F_{3 k+2}^{2}+F_{3 k+5}^{2}-F_{3 k-1}^{2}+15}{\left(F_{3 k+2}^{2}-F_{3 k-1}^{2}-1\right) F_{3 k+2}^{2}\left(F_{3 k+5}^{2}-F_{3 k+2}^{2}-1\right)} \\
& \quad>0,
\end{aligned}
$$

where the last inequality follows from the fact that $F_{3 k+5}=3 F_{3 k+2}+2 F_{3 k+1}$.

Therefore, we obtain

$$
\sum_{k=n}^{m n} \frac{1}{F_{3 k+2}^{2}}<\frac{1}{F_{3 n+2}^{2}-F_{3 n-1}^{2}-1}-\frac{1}{F_{3 m n+5}^{2}-F_{3 m n+2}^{2}-1}<\frac{1}{F_{3 n+2}^{2}-F_{3 n-1}^{2}-1}
$$

Applying (4.4) and the fact that $n$ is odd, we derive that

$$
\begin{aligned}
\sum_{k=n}^{m n} \frac{1}{F_{3 k+2}^{2}=} & \frac{1}{F_{3 n+2}^{2}-F_{3 n-1}^{2}}-\frac{1}{F_{3 m n+5}^{2}-F_{3 m n+2}^{2}} \\
& +\sum_{k=n}^{m n} \frac{(-1)^{k-1} 8 F_{3 k+2}^{2}-16}{\left(F_{3 k+2}^{2}-F_{3 k-1}^{2}\right) F_{3 k+2}^{2}\left(F_{3 k+5}^{2}-F_{3 k+2}^{2}\right)} \\
> & \frac{1}{F_{3 n+2}^{2}-F_{3 n-1}^{2}}+\frac{8 F_{3 n+2}^{2}-16}{\left(F_{3 n+2}^{2}-F_{3 n-1}^{2}\right) F_{3 n+2}^{2}\left(F_{3 n+5}^{2}-F_{3 n+2}^{2}\right)} \\
& -\frac{8 F_{3 n+5}^{2}+16}{\left(F_{3 n+5}^{2}-F_{3 n+2}^{2}\right) F_{3 n+5}^{2}\left(F_{3 n+8}^{2}-F_{3 n+5}^{2}\right)}-\frac{1}{F_{6 n+5}^{2}-F_{6 n+2}^{2}} \\
> & \frac{1}{F_{3 n+2}^{2}-F_{3 n-1}^{2}}+\frac{2}{F_{3 n+2}^{2}\left(F_{3 n+5}^{2}-F_{3 n+2}^{2}\right)}-\frac{2}{F_{3 n+5}^{2}\left(F_{3 n+5}^{2}-F_{3 n+2}^{2}\right)} \\
& -\frac{1}{F_{6 n+5}^{2}-F_{6 n+2}^{2}} \\
= & \frac{1}{F_{3 n+2}^{2}-F_{3 n-1}^{2}}+\frac{2}{F_{3 n+2}^{2} F_{3 n+5}^{2}}-\frac{1}{F_{6 n+5}^{2}-F_{6 n+2}^{2}} \\
> & \frac{1}{F_{3 n+2}^{2}-F_{3 n-1}^{2}}, \\
& \\
& \\
&
\end{aligned}
$$

where the last inequality follows from Lemma 4.1.

It follows from (4.7) and (4.8) that

$$
\frac{1}{F_{3 n+2}^{2}-F_{3 n-1}^{2}}<\sum_{k=n}^{m n} \frac{1}{F_{3 k+2}^{2}}<\frac{1}{F_{3 n+2}^{2}-F_{3 n-1}^{2}-1}
$$

which yields the desired result. 


\section{Conclusions}

In this paper, we investigate in two ways the reciprocal sums of the Fibonacci 3-subsequences, where a Fibonacci 3 -subsequence is a subsequence of the type $F_{n}, F_{n+3}, F_{n+6}, \ldots$. One is focused on the ordinary sums, and the other is concerned with the square sums. By evaluating the integer parts of the reciprocals of these sums we get several interesting families of identities. The results are new and important to those with closely related research interests.

Competing interests

The authors declare that they have no competing interests.

Authors' contributions

All authors contributed equally to deriving all the results of this article and read and approved the final manuscript.

\section{Acknowledgements}

The authors would like to thank the anonymous reviewers for their helpful comments. This work was supported by the National Natural Science Foundation of China (No. 11401080).

Received: 7 December 2015 Accepted: 18 January 2016 Published online: 26 January 2016

\section{References}

1. Duncan, RL: Applications of uniform distribution to the Fibonacci numbers. Fibonacci Q. 5, 137-140 (1967)

2. Holliday, SH, Komatsu, T: On the sum of reciprocal generalized Fibonacci numbers. Integers 11A, Article ID 11 (2011)

3. Karaduman, E: An application of Fibonacci numbers in matrices. Appl. Math. Comput. 147, $903-908$ (2004)

4. Koshy, T: Fibonacci and Lucas Numbers with Applications. Wiley, New York (2001)

5. Vorobiev, NN: Fibonacci Numbers. Springer, Basel (2002)

6. Elsner, C, Shimomura, S, Shiokawa, I: Algebraic relations for reciprocal sums of Fibonacci numbers. Acta Arith. 130 37-60 (2007)

7. Elsner, C, Shimomura, S, Shiokawa, I: Algebraic relations for reciprocal sums of odd terms in Fibonacci numbers. Ramanujan J. 17, 429-446 (2008)

8. Elsner, C, Shimomura, S, Shiokawa, I: Algebraic independence results for reciprocal sums of Fibonacci numbers. Acta Arith. 148, 205-223 (2011)

9. Elsner, C, Shimomura, S, Shiokawa, I: Algebraic relations for reciprocal sums of even terms in Fibonacci numbers. J. Math. Sci. 180, 650-671 (2012)

10. Ohtsuka, H, Nakamura, S: On the sum of reciprocal Fibonacci numbers. Fibonacci Q. 46/47, 153-159 (2008/2009)

11. Wang, AYZ, Wen, PB: On the partial finite sums of the reciprocals of the Fibonacci numbers. J. Inequal. Appl. 2015 Article ID 73 (2015)

12. Wang, AYZ, Zhang, F: The reciprocal sums of even and odd terms in the Fibonacci sequence. J. Inequal. Appl. 2015, Article ID 376 (2015)

\section{Submit your manuscript to a SpringerOpen ${ }^{\circ}$ journal and benefit from:}

- Convenient online submission

Rigorous peer review

- Immediate publication on acceptance

- Open access: articles freely available online

- High visibility within the field

- Retaining the copyright to your article 